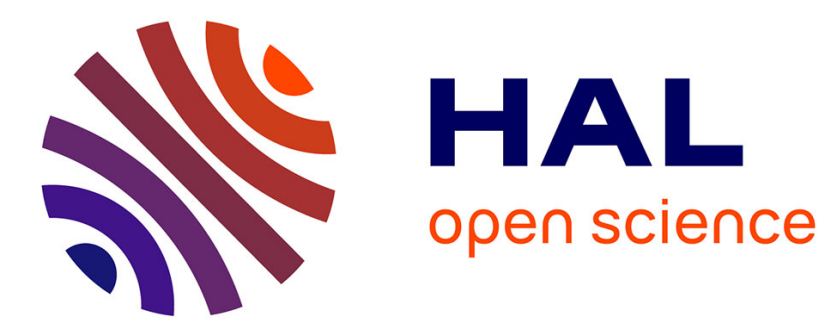

\title{
Element-resolved ultrafast demagnetization rates in ferrimagnetic CoDy
}

Tom Ferté, Nicolas Bergeard, L. Le Guyader, Michel Hehn, G. Malinowski, E. Terrier, E. Otero, K. Holldack, N. Pontius, Christine Boeglin

\section{- To cite this version:}

Tom Ferté, Nicolas Bergeard, L. Le Guyader, Michel Hehn, G. Malinowski, et al.. Element-resolved ultrafast demagnetization rates in ferrimagnetic CoDy. Physical Review B: Condensed Matter and Materials Physics (1998-2015), 2017, 96 (13), pp.134303 - 134303. 10.1103/PhysRevB.96.134303 . hal-01624257

\section{HAL Id: hal-01624257 \\ https://hal.univ-lorraine.fr/hal-01624257}

Submitted on 26 Oct 2017

HAL is a multi-disciplinary open access archive for the deposit and dissemination of scientific research documents, whether they are published or not. The documents may come from teaching and research institutions in France or abroad, or from public or private research centers.
L'archive ouverte pluridisciplinaire HAL, est destinée au dépôt et à la diffusion de documents scientifiques de niveau recherche, publiés ou non, émanant des établissements d'enseignement et de recherche français ou étrangers, des laboratoires publics ou privés. 


\title{
Element-resolved ultrafast demagnetization rates in ferrimagnetic CoDy
}

\author{
T. Ferté, ${ }^{1}$ N. Bergeard, ${ }^{1}$ L. Le Guyader,,${ }^{2,}{ }^{*}$ M. Hehn, ${ }^{3}$ G. Malinowski,${ }^{3}$ E. Terrier,,${ }^{1}$ E. Otero, ${ }^{4}$ \\ K. Holldack, ${ }^{2}$ N. Pontius, ${ }^{2}$ and C. Boeglin ${ }^{1}$ \\ ${ }^{1}$ Université de Strasbourg, CNRS, Institut de Physique et Chimie des Matériaux de Strasbourg, UMR 7504, F-67000 Strasbourg, France \\ ${ }^{2}$ Institut für Methoden und Instrumentierung der Forschung mit Synchrotronstrahlung, \\ Helmholtz-Zentrum Berlin für Materialien und Energie GmbH, Albert-Einstein-Strasse 15, 12489 Berlin, Germany \\ ${ }^{3}$ Institut Jean Lamour, Université Henri Poincaré, Nancy, France \\ ${ }^{4}$ Synchrotron SOLEIL, L'Orme des Merisiers, Saint-Aubin, 91192 Gif-sur-Yvette, France
}

(Received 7 March 2017; revised manuscript received 18 September 2017; published 9 October 2017)

\begin{abstract}
Femtosecond-laser-induced ultrafast magnetization dynamics have been studied in multisublattice $\mathrm{Co}_{x} \mathrm{Dy}_{1-x}$ alloys. By performing element- and time-resolved x-ray spectroscopy, we distinguish the ultrafast quenching of Co $3 d$ and Dy $4 f$ magnetic order when the initial temperatures are below $(T=150 \mathrm{~K})$ or above $(T=270 \mathrm{~K})$, the temperature of magnetic compensation $\left(T_{\text {comp }}\right)$. In accordance with former element-resolved investigations and theoretical calculations, we observe different dynamics for Co $3 d$ and Dy $4 f$ spins. In addition, we observe that for a given laser fluence, the demagnetization amplitudes and demagnetization times are not affected by the existence of a temperature of magnetic compensation. However, our experiment reveals a twofold increase of the ultrafast demagnetization rates for the Dy sublattice at low temperature. In parallel, we measure a constant

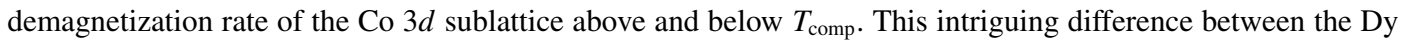
$4 f$ and Co $3 d$ sublattices calls for further theoretical and experimental investigations.
\end{abstract}

DOI: 10.1103/PhysRevB.96.134303

\section{INTRODUCTION}

Ultrafast demagnetization induced by infrared femtosecond laser pulses in magnetic layers was discovered 20 years ago [1]. Despite intensive theoretical [2-5] and experimental [6-8] works, a comprehensive and unified model describing the ultrafast demagnetization and the transfer of the angular momentum at the subpicosecond time scale is still missing. A broad range of systems have drawn attention over those two decades, including metallic transition metals [1,9-11], 4f lanthanides [12-16], semiconductors [17], half metals [18], and oxides [19]. Since the discovery of all-optical switching (AOS) by femtosecond infrared (IR) laser pulses [20], laser-induced magnetization dynamics in rare-earthtransition metal (RE-TM) alloys have received legitimate interest $[7,14,15,21-23]$. In these ferrimagnetic alloys, the TM $3 d$ itinerant and the RE $4 f$ localized magnetic moments are antiferromagnetically coupled through the RKKY (RudermanKittel-Kasuya-Yosida) indirect exchange mechanism [24,25]. For some RE-TM concentrations, it is possible to define a temperature where the magnetization of the two sublattices compensates each other's, resulting in a zero net total magnetization. Such specific temperature is named the temperature of magnetic compensation $T_{\text {comp }}$ [26-32] and is a macroscopic property of the RE-TM system. If laser-induced spin dynamics would be driven by microscopic parameters only, then $T_{\text {comp }}$ would not be expected to affect the dynamics. This is, however, in contradiction with Medapalli et al., who studied laser-induced ultrafast demagnetization in RE-TM alloys as a function of temperature below and above $T_{\text {comp }}[33,34]$. They showed that the quenching of the $3 d$ magnetic order is stronger when the initial temperature $T$ of the alloy is

\footnotetext{
*Present address: Spectroscopy \& Coherent Scattering, European XFEL GmbH, Holzkoppel 4, 22869 Schenefeld, Germany.
}

below $T_{\text {comp }}$. However, they did not monitor the RE $4 f$ spin dynamics [35]. During the same period, Lopez-Flores et al. reported different characteristic demagnetization times of the RE $4 f$ sublattice for initial temperatures below and above $T_{\text {comp }}$ [14]. In this latter work, a slower demagnetization observed for $T>T_{\text {comp }}$ has been attributed to a critical slowing down of the excited $4 f$ magnetic moments near the Curie temperature $\left(T_{\mathrm{C}}\right)$, rather than an effect of $T_{\text {comp }}$ itself. Surprisingly, the authors did not observe such a critical slowing down for the $3 d$ magnetic moments. Unfortunately, different RE-TM alloys with different magnetic properties (different RE elements, magnetic moments, anisotropies) were compared in this work. The interpretation given by Lopez-Flores et al. seems, however, in contradiction with recent theoretical results based on a Landau-Lifshitz-Bloch (LLB) model [36-41]. This model predicts that in RE-TM alloys, only the $3 d$ spins undergo a critical slowing down in the vicinity of $T_{\mathrm{C}}$. Therefore, a comprehensive element-resolved magnetization dynamics study across the compensation temperature is lacking.

In this publication, we address specifically the laser-induced ultrafast demagnetization dynamics in the vicinity of $T_{\text {comp }}$. We aim at extending the investigation of Medapalli et al. to the RE $4 f$ demagnetization rates, in order to reveal ultrafast spin dynamics in the vicinity of $T_{\text {comp }}[33,34,42]$. To do this we studied the ultrafast demagnetization rates of the Dy $4 f$ and Co $3 d$ magnetic sublattices in two CoDy alloys with identical static magnetic properties at the atomic scale (such as atomic magnetic moments and magnetic anisotropies), as verified in this work. Element-specific and time-resolved $\mathrm{X}$-ray magnetic circular dichroism (tr-XMCD) measurements were carried out at temperatures $T$ below and above $T_{\text {comp }}$ for a given laser fluence, allowing for an independent characterization of both sublattice demagnetization times and amplitudes [22,14,15,43]. Our results reveal important differences with those from Medapalli et al. [33,34]. We observed very close demagnetization amplitudes and characteristic 
demagnetization times for the Co sublattice for initial temperatures above and below $T_{\text {comp }}[33,34]$. Furthermore, we show that demagnetization amplitudes and characteristic demagnetization times are also similar in the case of the Dy sublattice. These results confirm that the temperature of magnetic compensation has no influence on these parameters. However, since the magnetization of the Dy sublattice is twice

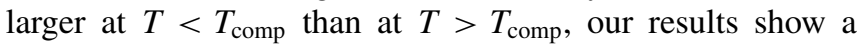
twofold increase of the ultrafast demagnetization rate of the Dy $4 f$ sublattice at low temperature. We show that in these CoDy alloys, none of the existing theoretical model is able to reproduce our experimental observations. Throughout this publication we aim at encouraging a theoretical description of laser-induced ultrafast dynamics, taking into account the temperature of magnetic compensation.

\section{EXPERIMENT}

Time-resolved x-ray magnetic circular dichroism operated in the "femtoslicing" mode at BESSY II $[34,10,35,36]$ is the most suitable technique to extract the ultrafast magnetization dynamics in multisublattice alloys, combining the magnetic and elemental sensitivity $[11,12,22,14,15,43]$. Femtosecond infrared laser pulses (with a wavelength of $800 \mathrm{~nm}$, linearly polarized with a 60 -fs pulse length) were used as the pump while the circularly polarized x-ray pulses with 100-fs duration were used as the probe [44]. The laser and x-ray spot size diameter at the sample location were approximately $500 \mu \mathrm{m}$ and $150 \mu \mathrm{m}$, respectively, which ensured optimal spatial overlap. The laser fluence was set to a constant value of $\mathrm{f}=7 \mathrm{~mJ} / \mathrm{cm}^{2}$, which ensured $\sim 60 \%$ demagnetization at the Co $L_{3}$ edge $[14,15]$. The repetition rate of the pump-probe experiment was $3 \mathrm{kHz}$, and the system relaxed toward its initial state between two subsequent laser excitations. The beamline energy was set to the Co $L_{3}$ or the Dy $M_{5}$ absorption edges. XMCD was monitored by recording the transmitted $\mathrm{X}$-ray intensities for opposite magnetic fields $(H= \pm 0.55 \mathrm{~T})$ as a function of the delay between the pump and the probe. The magnetic field was applied in the direction defined by the $\mathrm{x}$-ray beam.

The choice of the $\mathrm{Co}_{1-x} \mathrm{Dy}_{x}$ samples was motivated by the limitations of the experimental setup in terms of accessible temperatures and magnetic fields. Even by considering a very broad range of concentrations and RE elements, we were not able to determine a single TM-RE system with a moderate coercive field $\left(\mathbf{H}_{\mathrm{C}}\right)$ below $0.55 \mathrm{~T}$, below and above $T_{\text {comp }}$, in the limited temperature range of $80 \mathrm{~K}<T_{\text {comp }}<320 \mathrm{~K}$ $[27,28]$. Therefore we measured two different alloys, $\mathrm{Co}_{78} \mathrm{Dy}_{22}$ and $\mathrm{Co}_{80} \mathrm{Dy}_{20}$, with $T_{\text {comp }}=320 \mathrm{~K}$ and $220 \mathrm{~K}$, respectively. It is worth noting that in the framework of the mean field approximation (MFA), the microscopic magnetic properties such as the atomic magnetic moment $\left(\mu_{\mathrm{i}}\right)$ or the exchange coupling constant $\left(J_{\mathrm{ij}}\right)$ are considered independent of the composition and temperature [45]. Calculations based on MFA reproduced accurately the dependence of magnetic properties ( $T_{\text {comp }}, T_{\mathrm{C}}$, magnetization $M$, and $H_{\mathrm{c}}$ ) on composition and temperature [26-28,46]. Therefore, although our samples display disparate macroscopic properties $\left(H_{\mathrm{c}}, M, T_{\text {comp }}\right)$, they can be considered similar at the microscopic scale. We confirmed this assumption by static $\mathrm{x}$-ray transmission
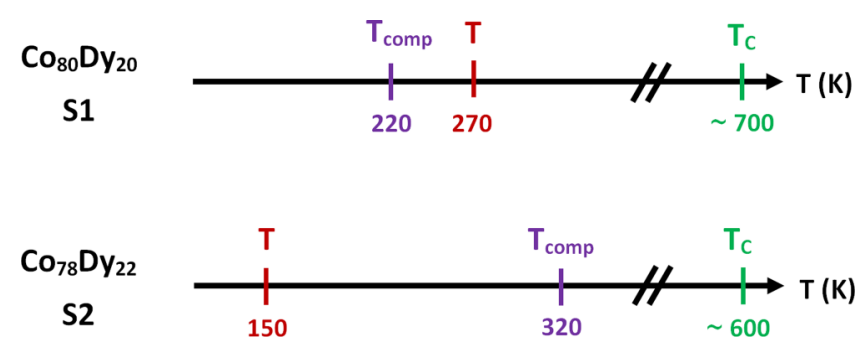

FIG. 1. Diagram of the characteristic temperatures for S1 and S2. $T$ is the temperature of the sample at negative delay (initial temperature) during the pump-probe experiment. $T_{\text {comp }}$ is the temperature of the magnetic compensation and $T_{\mathrm{C}}$ the Curie temperature of the alloys. The values of $T_{\mathrm{C}}$ are from Hansen et al. [27].

spectroscopy and XMCD measurements (see next section for details). The $\mathrm{Co}_{78} \mathrm{Dy}_{22}$ alloy displays $H_{\mathrm{c}}<0.55 \mathrm{~T}$ at $T<150 \mathrm{~K}$, while the $\mathrm{Co}_{80} \mathrm{Dy}_{20}$ alloy displays $H_{\mathrm{c}}<0.55 \mathrm{~T}$ at $T>270 \mathrm{~K}$. The low magnetic coercive fields of the alloys ensure magnetic saturation under the applied $+/-0.55 \mathrm{~T}$ magnetic field available on the FEMTOSPEX end station. In addition, the experiment can be performed at temperatures below $300 \mathrm{~K}$, as annealing effects have been observed for other sample compositions at higher temperature. Following our static sample characterization we can safely compare the ultrafast dynamics measured for both samples. In the rest of the article, we define the following names S1 and S2 corresponding to $\mathrm{Co}_{80} \mathrm{Dy}_{20}$ and $\mathrm{Co}_{78} \mathrm{Dy}_{22}$, respectively, for which the laser-induced dynamics was monitored at $T>T_{\text {comp }}$ and $T<T_{\text {comp }}$, respectively, as illustrated in Fig. 1.

The 18-nm-thick CoDy alloys were sputter-deposited on X-ray transparent 200-nm-thick $\mathrm{Si}_{3} \mathrm{~N}_{4}$ membranes and capped with a 3-nm Ta layer to prevent oxidation. A heat sink and buffer layer composed of 80-nm-thick Ta/Cu multilayers were grown between the alloys and the membranes. This buffer layer limits the temperature increase due to laser dc heating (estimated) to $\sim 70 \mathrm{~K}$. The total $\mathrm{x}$-ray transmission of the stacks in the vicinity of the Co and Dy absorption edges was about $50 \%$. The dependence of the alloy magnetization with temperature was measured by vibrating sample magnetometersuperconducting quantum interference device (VSM-SQUID) to determine the temperature of magnetic compensation $T_{\text {comp }}$ of our CoDy alloys. This temperature is also an accurate indication of the effective composition, since a tiny change in the concentration induces a large variation in $T_{\text {comp }}[27,28]$.

The static XMCD spectroscopy was performed at the DEIMOS beamline at synchrotron SOLEIL in order to characterize the magnetic properties of the alloys. The measurements were performed in transmission and at normal incidence, i.e., the same geometry as the one used in the pump-probe experiments at the "femtoslicing" beamline. The x-ray transmission spectra were recorded under a $\pm 2 \mathrm{~T}$ magnetic field applied parallel to the $\mathrm{x}$-ray beam for both left- and right-circular polarizations.

\section{EXPERIMENTAL RESULTS}

\section{A. Magnetic properties}

In Fig. 2(a) we show the X-ray transmission signals measured at the Dy $M_{5}$ edge at $T=150 \mathrm{~K}$ and negative 

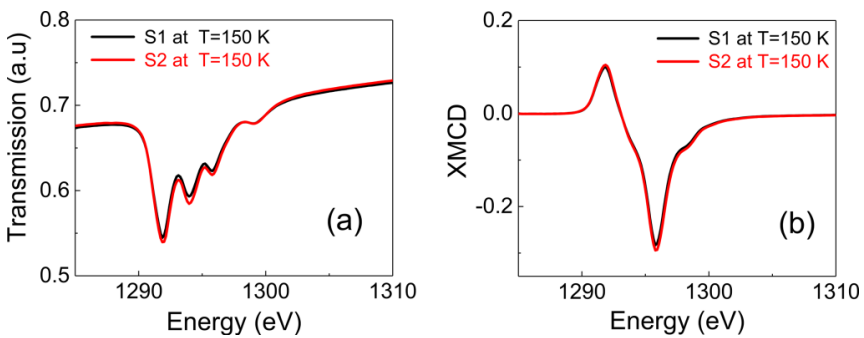

FIG. 2. (a) X-ray transmission signals measured for negative helicity at the Dy $M_{5}$ edge for $\mathrm{Co}_{80} \mathrm{Dy}_{20}$ (S1) (black line) and for $\mathrm{Co}_{78} \mathrm{Dy}_{22}$ (S2) at $T=150 \mathrm{~K}$ (red line). At $T=150 \mathrm{~K}$, the similarity of the multiplet structures shows that both alloys can be assumed to be identical from the point of view of the electronic structure. (b) XMCD at the Dy $M_{5}$ edges for $\mathrm{Co}_{80} \mathrm{Dy}_{20}(\mathrm{~S} 1)$ (black line) and $\mathrm{Co}_{78} \mathrm{Dy}_{22}(\mathrm{~S} 2)$ at $150 \mathrm{~K}$ (red line). The similarity of the XMCD amplitudes and shape at $T=150 \mathrm{~K}$ shows that both alloys can be assumed identical from the point of view of the magnetic moments.

helicity for S1 and S2. At $T=150 \mathrm{~K}$ both alloys (S1 and S2) are characterized by the same multiplet structures and thus the same occupation of the Zeeman levels [47]. In addition, the static XMCD spectra recorded at the Dy $M_{5}$ edge are compared for both samples in Fig. 2(b) and show identical features at $T=150 \mathrm{~K}$. The similarities of the multiplet structures and XMCD signals observed at $T=150 \mathrm{~K}$ ensure very close electronic and magnetic properties for S1 and S2. In Figs. 3(a) and 3(b) we show the transmission signal measured for positive and negative x-ray helicities, recorded at $150 \mathrm{~K}$ at the $\mathrm{Co} L_{2,3}$ and Dy $M_{4,5}$ edges for S1 and S2. The sum rules analysis, including the spin dipolar moments, was used in order to extract the magnetic moments per atoms $m_{i}$ of Co and Dy in both alloys (Table I) [48-51]. Here, $m_{i}$ is defined as the sample-averaged projection along the x-ray incidence of the individual atomic magnetic moments $\mu_{\mathrm{i}}$. These values, which are listed in Table I, show that the magnetic moments of Co and Dy are consistent with literature [47,52-54] and they confirm that at $T=150 \mathrm{~K}$, both alloys have the same magnetic moments within the error bars. We can thus safely compare the ultrafast magnetization dynamics below and above $T_{\text {comp }}$, comparing the data measured for S1 and S2.

The thermal dependences of the magnetic moments in Co and Dy and the coercive fields have been analyzed in the temperature range used during the pump-probe experiments $(150-270 \mathrm{~K})$. In Fig. 4 we show the XMCD at Dy $M_{5}$ for both alloys at temperatures of $150 \mathrm{~K}$ and $270 \mathrm{~K}$. The
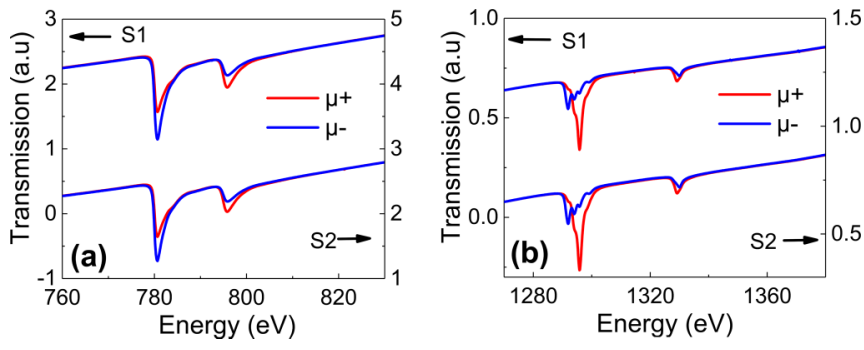

FIG. 3. X-ray transmission signal measured at $T=150 \mathrm{~K}$ for positive $\left(\mu^{+}\right)$and negative $\left(\mu^{-}\right)$helicities at the (a) Co $L_{2,3}$ and (b) Dy $M_{4,5}$ edges for $\mathrm{Co}_{80} \mathrm{Dy}_{20}(\mathrm{~S} 1)$ and $\mathrm{Co}_{78} \mathrm{Dy}_{22}$ (S2). The spectra are normalized such as $\left(\mu^{+}+\mu^{-}\right) / 2=1$ at the $\operatorname{Co} L_{3}$ and Dy $M_{5}$ edges.

inset shows the hysteresis curves extracted from the Dy $M_{5}$ XMCD data as a function of the applied field. The loops are square for both alloys $\mathrm{S} 1$ and $\mathrm{S} 2$ and confirm the limited coercive fields $\left(H_{\mathrm{C}}<0.55 \mathrm{~T}\right)$. In Table I we can see that the Dy sublattice shows a lower magnetic moment at $T=270 \mathrm{~K}\left(m=-4.12 \pm 0.21 \mu_{\mathrm{B}} /\right.$ at $)$ than at $T=150 \mathrm{~K}$ ( $\left.m=+6.64 \pm 0.33 \mu_{\mathrm{B}} / \mathrm{at}\right)$, whereas the magnetic moment of Co does not change within the error bars. The quantitative magnetic moments measured for Co and Dy, as well as their thermal dependences, are consistent with experimental values obtained for similar alloys [47,55]. At $T=270 \mathrm{~K}$ (compared to $150 \mathrm{~K}$ ) the low Dy $M_{5} \mathrm{XMCD}$ signal results from the large thermal fluctuations affecting the Zeeman levels. Therefore we can conclude that the temperature dependence of the Dy $M_{5}$ XMCD signal is not influenced by $T_{\text {comp }}$ [46]. Our quantitative element-resolved values of the magnetic moments will be used in the next section. They are essential ingredients to derive the demagnetization rates (D) in each sublattice, representing the ability of the Co and Dy spins to transfer the angular momenta to other subsystems as, for instance, the lattice.

\section{B. Ultrafast magnetization dynamics in CoDy alloys by tr-XMCD}

The time-resolved XMCD signal recorded over short delay ranges ( $\sim 4 \mathrm{ps}$ ) at the Co $L_{3}$ and Dy $M_{5}$ edges are displayed in Figs. 5(a) and 5(b), respectively. At negative delays $t=t^{<}$, the XMCD signals at the Dy $M_{5}$ edge are proportional to the static XMCD determined at similar temperatures in Table I. Over the whole investigated delay range, the sign of the XMCD signal remains constant, excluding any laser-induced magnetization reversal [22]. However, different demagnetization amplitudes

TABLE I. Static spin (m spin), orbital (m orb), and total (m) magnetic moments for Co and Dy obtained by applying the sum rules [48,49]. The XMCD experiments were performed at the Co $L_{2,3}$ and Dy $M_{4,5}$ edges at $T=150 \mathrm{~K}$ for S1 and S2 and at $T=270 \mathrm{~K}$ for S1. We show that both alloys have identical magnetic moments at $T=150 \mathrm{~K}$ within the error bars.

\begin{tabular}{lccrrr}
\hline \hline Sample & $T(\mathrm{~K})$ & Element & m spin $\left(\mu_{\mathrm{B}} / \mathrm{at}\right)$ & m orb $\left(\mu_{\mathrm{B}} / \mathrm{at}\right)$ & m $\left(\mu_{\mathrm{B}} / \mathrm{at}\right)$ \\
\hline $\mathrm{Co}_{80} \mathrm{Dy}_{20}$ & 150 & Co & $-1.50 \pm 0.08$ & $-0.19 \pm 0.02$ & $-1.69 \pm 0.10$ \\
$(\mathrm{~S} 1)$ & & Dy & $2.98 \pm 0.15$ & $3.66 \pm 0.18$ & $6.64 \pm 0.33$ \\
& 270 & Co & $1.66 \pm 0.08$ & $0.22 \pm 0.02$ & $-4.88 \pm 0.10$ \\
& & Dy & $-1.89 \pm 0.10$ & $-2.23 \pm 0.11$ & $-1.53 \pm 0.09$ \\
$\mathrm{Co}_{78} \mathrm{Dy}_{22}$ & 150 & $\mathrm{Co}$ & $-1.35 \pm 0.07$ & $-0.18 \pm 0.02$ & $6.55 \pm 0.33$ \\
$(\mathrm{~S} 2)$ & & Dy & $2.96 \pm 0.15$ & $3.59 \pm 0.18$ & \\
\hline \hline
\end{tabular}




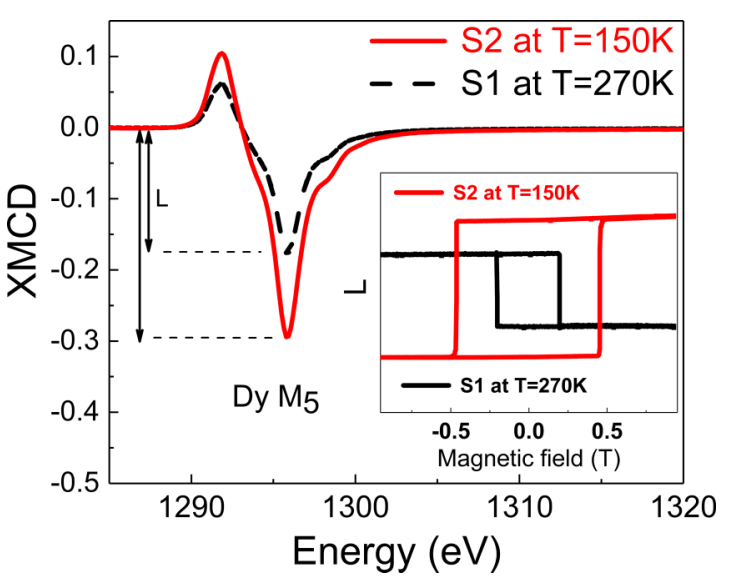

FIG. 4. XMCD at the Dy $M_{5}$ edge for $\mathrm{S} 1$ (black line) at $T=$ $270 \mathrm{~K}$ and $\mathrm{S} 2$ at $150 \mathrm{~K}$ (red line). The inset shows the magnetic hysteresis measured along the normal of the surface at the Dy $M_{5}$, defined by the XMCD amplitude $L$ as illustrated. The hysteresis obtained for both alloys is superposed. They are measured at the temperatures used during the pump-probe experiments $(270 \mathrm{~K}$ and $150 \mathrm{~K})$. At these temperatures the alloys show identical out-of-plane anisotropies with low coercive fields $\left(H_{\mathrm{C}}\right)$ below $0.55 \mathrm{~T}$.

as well as a different dynamic of magnetization recovery are visible between Co and Dy at both temperatures. The transient XMCD signal at the Co $L_{3}$ edge shows a relative demagnetization amplitude of $\sim 60 \%$, followed by the magnetization recovery. The transient XMCD signal at the Dy $M_{5}$ edge shows an almost completely quenched magnetization and no recovery. Furthermore, the Co magnetization reaches its minimum value $\left[m\left(t^{*}\right)\right]$ at a delay $t^{*} \sim 0.5 \mathrm{ps}$, for which the Dy magnetization achieved only half of its total demagnetization. The Dy magnetization reaches its minimum value $\left[m\left(t^{*}\right)\right]$ at a delay $t^{*} \approx 2$ ps. Transient normalized XMCD at Co $L_{3}$ and Dy $M_{5}$ edges is shown in Figs. 6(a) and 6(b) in order to compare the element-resolved ultrafast demagnetization at temperatures $T$ above and below $T_{\text {comp }}\left(T>T_{\text {comp }}\right.$ and $T<$ $\left.T_{\text {comp }}\right)$ in each subsystem. The curves are fits to the data with a single exponential decay followed by an exponential recovery. Longer delay scans (not shown) have been performed in order to estimate the recovery times (Table II). The fit function is convoluted by a Gaussian function representing the time resolution of the experiments $\sim 130 \mathrm{fs}[10,11]$. The relative demagnetization amplitudes $A=\left[m\left(t^{<}\right)-m\left(t^{*}\right)\right] / m\left(t^{<}\right)$as well as the characteristic demagnetization times $(\tau)$ and
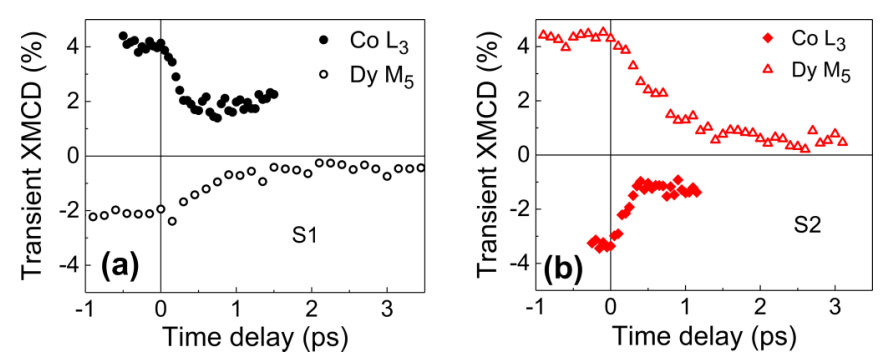

FIG. 5. Transient XMCD at the Co $L_{3}$ (squares) and Dy $M_{5}$ (circles) edges for the $\mathrm{S} 1$ (a) and the $\mathrm{S} 2$ (b) alloys as a function of the delay. The laser fluence was $7 \mathrm{~mJ} / \mathrm{cm}^{2}$.
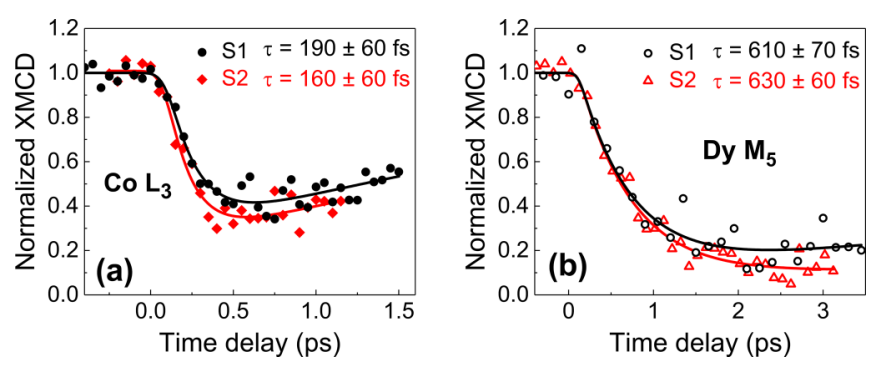

FIG. 6. Transient normalized XMCD at the Co $L_{3}$ (a) and Dy $M_{5}$ (b) edges for the $\mathrm{S} 1$ (black symbols) and the $\mathrm{S} 2$ (red symbols) alloys as a function of the delay. The solid lines are fits to the data by a double exponential convoluted by a Gaussian function. The laser fluence was $7 \mathrm{~mJ} / \mathrm{cm}^{2}$.

recovery times $\left(\tau_{\mathrm{R}}\right)$, extracted from the fit function, are reported in Table II. The ultrafast demagnetization rate $(D)$, defined as the ratio between the absolute demagnetization amplitude $A m\left(t^{<}\right)$and the characteristic demagnetization time $\tau$ [56-58],

$$
D=\left[m\left(t^{<}\right)-m\left(t^{*}\right)\right] / \tau\left(\text { in } \mu_{\mathrm{B}} / \mathrm{s} \text { at }\right),
$$

characterizes the ability of the spins to transfer the angular momentum away. The calculated values from our experimental data for the Co and Dy sublattices are shown in Table II.

For the Co sublattice, at $T<T_{\text {comp }}$ and $T>T_{\text {comp }}$, the laser-induced ultrafast relative demagnetization amplitudes $A$ are $59 \pm 5 \%$ and $65 \pm 4 \%$, and the demagnetization times $\tau$ are $190 \pm 60 \mathrm{fs}$ and $160 \pm 60 \mathrm{fs}$, respectively, as shown in Table II. Those values show that within the error bars, the extracted values $D$ are similar at both temperatures $(5.8 \pm 2.7$ and $6.2 \pm 3.1 \mu_{\mathrm{B}} / \mathrm{ps}$ at). For the Dy sublattice, at $T<T_{\text {comp }}$ and $T>T_{\text {comp }}$, the relative demagnetization amplitudes $A$ are $80 \pm 9 \%$ and $92 \pm 8 \%$ and the demagnetization times $\tau$ are $610 \pm 70 \mathrm{fs}$ and $630 \pm 60 \mathrm{fs}$, respectively. They also remain similar within our error bars. However, the extracted values $D$ are largely different at both temperatures $\left(9.6 \pm 2.2 \mu_{\mathrm{B}} / \mathrm{ps}\right.$ at and $5.4 \pm 1.5 \mu_{\mathrm{B}} / \mathrm{ps}$ at). This is a consequence of the difference between the Dy magnetization at $T<T_{\text {comp }}$ and $T>T_{\text {comp }}$ (Table II). Our results show that at $T<T_{\text {comp }}$, $D$ is nearly twice as large as at $T>T_{\text {comp. Moreover, in }}$ spite of the strong $3 d-4 f$ indirect exchange coupling, the Co and Dy sublattices show different relative demagnetization amplitudes (average values $\sim 60 \%$ and $\sim 85 \%$, respectively) and different characteristic demagnetization times (average values $\sim 170 \mathrm{fs}$ and $\sim 620 \mathrm{fs}$, respectively), consistent with the various element- and time-resolved XMCD experiments reported in literature $[14,15,22,43,59,60]$.

\section{DISCUSSION}

In a recent work, Medapalli et al. reported larger demagnetization amplitudes of the FeCo sublattice in $\mathrm{FeCoGd}$ alloys for initial temperatures below $T_{\text {comp }}[33,34]$. This is not observed for the Co sublattice in our CoDy alloys. We show in this work that the conclusions extracted from the FeCoGd alloys cannot be extended to our RE-TM alloys. It is worth noting that in FeCoGd alloys, experimental $[7,20-23]$ and theoretical $[41,42,61-64]$ results demonstrate that in addition to the 
TABLE II. Values of the relative demagnetization amplitude $A=\left[m\left(t^{<}\right)-m\left(t^{*}\right)\right] / m\left(t^{<}\right)$, characteristic demagnetization time $\tau$, and recovery time $\tau_{\mathrm{R}}$, extracted from the fit function of the experimental demagnetization dynamics. The ultrafast demagnetization rates $D=$ $\left[m\left(t^{<}\right)-m\left(t^{*}\right)\right] / \tau$ are calculated from the other quantities. The static magnetic moments $m\left(t^{<}\right)$are extracted from the sum rules analysis. All quantities are given for Co $3 d$ and Dy $4 f$ at $T=270 \mathrm{~K}$ and $150 \mathrm{~K}$.

\begin{tabular}{lcccrrrr}
\hline \hline Sample & & & & & & \multicolumn{2}{c}{$D=\left[m\left(t^{<}\right)-m\left(t^{*}\right)\right] / \tau$} \\
$\left(\mu_{\mathrm{B}} / \mathrm{ps}\right.$ at $)$
\end{tabular}

laser-induced demagnetization an additional phenomenon, namely, the helicity-independent all-optical switching (HIAOS) of the spins, is at play [65]. We note that this HI-AOS is only observed for sample temperature in the proximity of $T_{\text {comp }}$ [66]. Therefore, the enhanced demagnetization amplitudes in FeCoGd at $T<T_{\text {comp }}$ as reported by Medapalli et al. is probably a specificity of this alloy and a signature of HI-AOS $[42,65]$. It has been shown that the demagnetization amplitudes upon laser excitation are fluence [67-69] and temperature dependent $\left(T-T_{\mathrm{C}}\right.$ ) [70]. Therefore, we can safely conclude that the similar demagnetization amplitudes which are observed in our study at $T<T_{\text {comp }}$ and $T>T_{\text {comp }}$ are a consequence of the close values of our experimental parameters, laser fluences, and distance from $T_{\mathrm{C}}\left(\left|T-T_{\mathrm{C}}\right| \sim 450 \pm 20 \mathrm{~K}\right)$. Finally, our results further confirm that the macroscopic parameter $T_{\text {comp }}$ has no influence on the demagnetization amplitudes $m\left(t^{<}\right)-m\left(t^{*}\right)$.

Let us discuss the characteristic demagnetization times $(\tau)$ extracted for Co and Dy in Table II. In a former publication, we have shown that larger $\tau$ values could be measured for Tb in $\mathrm{Co}_{86} \mathrm{~Tb}_{14}(\tau \approx 500 \mathrm{fs})$ compared to $\mathrm{Co}_{74} \mathrm{~Tb}_{26}(\tau \approx 280 \mathrm{fs})$, and we related them to the proximity of the temperature to $T_{\mathrm{C}}\left(T-T_{\mathrm{C}}\right)$ [14]. These results are also confirmed by Atxitia et al. [61] based on the LLB model, which shows that $\tau$ scales with $1 /\left(T-T_{\mathrm{C}}\right)$ when $T$ is close to $T_{\mathrm{C}}$.

In this work, however, we use values of $\left|T-T_{\mathrm{C}}\right|$ close to $450 \mathrm{~K}$ during the experiments at $T<T_{\text {comp }}$ and $T>$

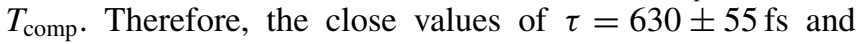
$\tau=610 \pm 65 \mathrm{fs}$ measured for Dy are consistent with our previous conclusions where $\tau$ depends on $\left|T-T_{\mathrm{C}}\right|$ [14]. In a recent work by Mentink et al., a phenomenological model was proposed in order to describe ultrafast spin dynamics in RETM alloys [71]. They show that in the temperature-dominated regime, for which the electronic temperature $\left(T_{\mathrm{e}}\right)$ is far above $T_{\mathrm{C}}, \tau$ is given by

$$
\tau=\mu /\left(2 \alpha \gamma k_{\mathrm{B}} T_{\mathrm{e}}\right),
$$

with $\mu$ the atomic magnetic moment (independent from the thermal fluctuations), $\alpha$ the coupling constant with the heat bath, $\gamma$ the gyromagnetic ratio, and $k_{\mathrm{B}}$ the Boltzmann's constant [36,72]. Radu et al. applied this phenomenological model to establish a linear relation between $\tau$ and $m$ with different RE-TM alloys [43]. In our work we can assume an equivalent elevation of the electronic temperature $T_{\mathrm{e}}$ upon laser excitations for S1 and S2, which is guaranteed by the very same laser fluence used for both experiments. Assuming that the parameters $\mu_{\mathrm{i}}, \alpha_{\mathrm{i}}$, and $\gamma_{\mathrm{i}}$ are similar for S1 and S2, we can stress that the phenomenological model from Mentink et al. supports our results and justifies the similarity of $\tau$ at $T<T_{\text {comp }}$ and $T>T_{\text {comp }}$.

Several other theoretical models, such as the LLB model [39-41,61] and the microscopic three-temperature model (m3TM) [3,63] have related $\tau$ to microscopic magnetic properties of ferrimagnets. These models predict that the macroscopic parameter $T_{\text {comp }}$ has no influence on $\tau$, in line with our results. Our conclusions are further sustained by recent work from Rettig et al. for the case of antiferromagnetic Ho layers [16]. They showed that for Ho layers without finite magnetization, $\tau$ is similar to those values reported for ferromagnetic $\mathrm{Gd}$ and $\mathrm{Tb}$ [12]. They evidenced that a zero net magnetization in antiferromagnetically coupled sublattices does not influence the demagnetization time.

In conclusion, we show that the $T_{\text {comp }}$ value has no influence on the demagnetization amplitude and characteristic demagnetization times. This assumption is supported by phenomenological models assuming that these parameters are determined by the microscopic magnetic properties of our alloys while $T_{\text {comp }}$ is a macroscopic parameter.

So far, we have qualitatively discussed both features defined by $\tau$ and by the amplitude of demagnetization for Co and Dy at temperatures $T$ above and below $T_{\text {comp }}$. These quantities are used to define the demagnetization rates $D$ characterizing the ability of the spin system to transfer the angular momenta to other subsystems. The extracted demagnetization rates $D$ for Co are $6.2 \pm 3.1$ and $5.8 \pm 2.7 \mu_{\mathrm{B}} / \mathrm{ps}$ at for $T<T_{\text {comp }}$ (sample S2) and $T>T_{\text {comp }}$ (sample $\mathrm{S} 1$ ), respectively (Table II). For the Dy sublattice, we observe a twofold increase of the demagnetization rate $D$ at $T<T_{\text {comp }}\left(D=9.6 \pm 2.2 \mu_{\mathrm{B}} / \mathrm{ps}\right.$ at $)$ compared to $T>T_{\text {comp }}\left(D=5.4 \pm 1.5 \mu_{\mathrm{B}} / \mathrm{ps}\right.$ at) (Table II). Comparing all those numbers, we observe very close values of $D$ except for the Dy sublattice at $T<T_{\text {comp }}$.

In the framework of the LLB model, the demagnetization rates of both $\mathrm{Fe}$ and $\mathrm{Gd}$ sublattices in $\mathrm{FeCoGd}$ alloy scale with their respective magnetization (Eq. (10) in [61]). This model should in principle reproduce our experimental observations. However, the LLB equation describes pure spin dynamics, neglecting the contribution of the orbital moments to the magnetization, which is large for Dy and essentially zero for Gd. Therefore, the description of the laser-induced demagnetization of the Dy sublattice in CoDy alloys may not be straightforward by using the LLB model [73]. Recently, Donges et al. have derived the 
Landau-Lifshitz-Gilbert (LLG) equation to describe accurately the thermal magnetic properties of CoDy alloys [55]. Unfortunately, the authors have not extended their calculation to laser-induced ultrafast dynamics yet. By comparing with literature, we can further support such temperature dependence of $D$ for Dy and other RE elements in RE-TM alloys. Radu et al. reported quantitative element-resolved investigation of laser-induced ultrafast demagnetization in a $\mathrm{Co}_{83} \mathrm{Dy}_{17}$ alloy, measured at $T>T_{\text {comp [43]. We derived very close values of }}$ $D$ for both Co and Dy sublattices: $D=4.1 \pm 1.6 \mu_{\mathrm{B}} / \mathrm{ps}$ at and $D=4.2 \pm 1.3 \mu_{\mathrm{B}} / \mathrm{ps}$ at, respectively [43]. Furthermore, different values of $D$ measured at different temperatures $T>T_{\text {comp }}$ and $T<T_{\text {comp }}$ can be extracted from the work of Lopez et al. in CoTb alloys [14]. The Tb sublattice shows the following values: $D=6.12 \pm 3.2 \mu_{\mathrm{B}} / \mathrm{ps}$ at for $\mathrm{Co}_{86} \mathrm{~Tb}_{14}$ measured at $T>T_{\text {comp }}$ and $D=9.7 \pm 2.3 \mu_{\mathrm{B}} / \mathrm{ps}$ at

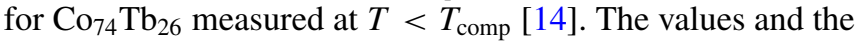
increase of $D$ at low temperature are coherent with the values we extracted for the Dy sublattice in CoDy alloys, suggesting that the effect is not a specificity of CoDy. All those values indicate a more efficient transfer of the angular momenta below $T_{\text {comp }}$ than above $T_{\text {comp }}$ for RE sublattices in RE-TM alloys.

Theoretical models have predicted that the proximity of $T_{\text {comp }}$ could have an influence on laser-induced dynamics in RE-TM alloys. Gridnev predicted recently that the exchange scattering should be more effective for $T<T_{\text {comp }}$ [42]. Barker et al. demonstrated that the magnon dispersions are dependent on the composition, and thus on the net magnetization, of the alloys [74]. Wienholdt et al. predicted spin dynamics in the $\mathrm{THz}$ regime in the vicinity of $T_{\text {comp }}$ [32]. However, these models are focused on laser-induced dynamics in FeCoGd alloys where the RE orbital magnetic moment is zero $[33,34]$. Throughout this work, we aim at motivating additional experimental investigations and theoretical descriptions of laser-induced dynamics in the vicinity of $T_{\text {comp }}$ in other RE-TM alloys than the FeCoGd alloys.

\section{CONCLUSIONS}

We investigated the laser-induced ultrafast magnetization dynamics with element selectivity in $\mathrm{Co}_{78} \mathrm{Dy}_{22}$ and $\mathrm{Co}_{80} \mathrm{Dy}_{20}$ alloys with initial temperatures below $(T=150 \mathrm{~K})$ and above $(T=270 \mathrm{~K}) T_{\text {comp }}$. We combined static and time-resolved XMCD spectroscopies in order to derive quantitative values for the demagnetization rates for each element and temperature. We demonstrated that the demagnetization amplitude and characteristic demagnetization times, determined by microscopic parameters, are not influenced by $T_{\text {comp }}$. The demagnetization rates are the same within the error bars for Co at both initial temperatures and for Dy at $T>T_{\text {comp }}$, while below $T_{\text {comp }}$ we observe a twofold increase in the demagnetization rate of Dy. It is not clear whether this enhanced demagnetization rate is induced by macroscopic properties of the alloys such as $T_{\text {comp }}$. These measurements hold appeal for complementary experimental investigations at different temperatures, elements, and laser fluences. We also hope these quantitative data will motivate further theoretical works in order to identify the role, or lack thereof, of the temperature of compensation on laserinduced ultrafast demagnetization in ferrimagnetic alloys.

\section{ACKNOWLEDGMENTS}

We are indebted to R. Mitzner and T. Kachel for help and support during the femtoslicing experiments. The authors are grateful for financial support received from the following agencies: The French "Agence National de la Recherche" via Projects No. ANR-11-LABX-0058_NIE and No. UMAMI ANR-15-CE24-0009, the EQUIPEX UNION through Project No. ANR-10-EQPX-52, the CNRS-PICS program, the European Community's Seventh Framework Programme (FP7/2007-2013) under Grant Agreement No. 281043 (FEMTOSPIN), and the EU Contract Integrated Infrastructure Initiative I3 within FP6 Project No. R II 3CT-2004-50600008. We acknowledge Synchrotron-SOLEIL for the provision of synchrotron radiation facilities.
[1] E. Beaurepaire, J.-C. Merle, A. Daunois, and J.-Y. Bigot, Phys. Rev. Lett. 76, 4250 (1996).

[2] G. P. Zhang and W. Hübner, Phys. Rev. Lett. 85, 3025 (2000).

[3] B. Koopmans, G. Malinowski, F. Dalla Longa, D. Steiauf, M. Fähnle, T. Roth, M. Cinchetti, and M. Aeschlimann, Nat. Mater. 9, 259 (2010).

[4] M. Battiato, K. Carva, and P. M. Oppeneer, Phys. Rev. Lett. 105, 027203 (2010).

[5] B. Y. Mueller, A. Baral, S. Vollmar, M. Cinchetti, M. Aeschlimann, H. C. Schneider, and B. Rethfeld, Phys. Rev. Lett. 111, 167204 (2013).

[6] A. Kirilyuk, A. V. Kimel, and T. Rasing, Rev. Mod. Phys. 82, 2731 (2010).

[7] A. Kirilyuk, A. V. Kimel, and T. Rasing, Rep. Prog. Phys. 76, 026501 (2013).

[8] J.-Y. Bigot and M. Vomir, Ann. Phys. 525, 2 (2013).

[9] E. Beaurepaire, M. Maret, V. Halté, J.-C. Merle, A. Daunois, and J.-Y. Bigot, Phys. Rev. B 58, 12134 (1998).
[10] C. Stamm, T. Kachel, N. Pontius, R. Mitzner, T. Quast, K. Holldack, S. Khan, C. Lupulescu, E. F. Aziz, M. Wiestruck et al., Nat. Mater. 6, 740 (2007).

[11] C. Boeglin, E. Beaurepaire, V. Halté, V. López-Flores, C. Stamm, N. Pontius, H. A. Dürr, and J.-Y. Bigot, Nature (London) 465, 458 (2010).

[12] M. Wietstruk, A. Melnikov, C. Stamm, T. Kachel, N. Pontius, M. Sultan, C. Gahl, M. Weinelt, H. A. Dürr, and U. Bovensiepen, Phys. Rev. Lett. 106, 127401 (2011).

[13] M. Sultan, U. Atxitia, A. Melnikov, O. ChubykaloFesenko, and U. Bovensiepen, Phys. Rev. B 85, 184407 (2012).

[14] V. López-Flores, N. Bergeard, V. Halté, C. Stamm, N. Pontius, M. Hehn, E. Otero, E. Beaurepaire, and C. Boeglin, Phys. Rev. B 87, 214412 (2013).

[15] N. Bergeard, V. López-Flores, V. Halté, M. Hehn, C. Stamm, N. Pontius, E. Beaurepaire, and C. Boeglin, Nat. Commun. 5, 3466 (2014). 
[16] L. Rettig, C. Dornes, N. Thielemann-Kühn, N. Pontius, H. Zabel, D. L. Schlagel, T. A. Lograsso, M. Chollet, A. Robert, M. Sikorski et al., Phys. Rev. Lett. 116, 257202 (2016).

[17] M. Battiato and K. Held, Phys. Rev. Lett. 116, 196601 (2016).

[18] G. M. Müller, J. Walowski, M. Djordjevic, G.-X. Miao, A. Gupta, A. V. Ramos, K. Gehrke, V. Moshnyaga, K. Samwer, J. Schmalhorst et al., Nat. Mater. 8, 56 (2009).

[19] C.-H. Hsia, T.-Y. Chen, and D. H. Son, Nano Lett. 8, 571 (2008).

[20] C. D. Stanciu, A. Tsukamoto, A. V. Kimel, F. Hansteen, A. Kirilyuk, A. Itoh, and T. Rasing, Phys. Rev. Lett. 99, 217204 (2007).

[21] K. Vahaplar, A. M. Kalashnikova, A. V. Kimel, D. Hinzke, U. Nowak, R. Chantrell, A. Tsukamoto, A. Itoh, A. Kirilyuk, and T. Rasing, Phys. Rev. Lett. 103, 117201 (2009).

[22] I. Radu, K. Vahaplar, C. Stamm, T. Kachel, N. Pontius, H. A. Dürr, T. A. Ostler, J. Barker, R. F. L. Evans, R. W. Chantrell et al., Nature (London) 472, 205 (2011).

[23] T. A. Ostler, J. Barker, R. F. L. Evans, R. W. Chantrell, U. Atxitia, O. Chubykalo-Fesenko, S. El Moussaoui, L. Le Guyader, E. Mengotti, L. J. Heyderman et al., Nat. Commun. 3, 666 (2012).

[24] M. S. S. Brooks, L. Nordström, and B. Johansson, J. Phys.: Condens. Matter 3, 2357 (1991).

[25] I. A. Campbell, J. Phys. F 2, L47 (1972).

[26] P. Hansen, C. Clausen, G. Much, M. Rosenkranz, and K. Witter, J. Appl. Phys. 66, 756 (1989).

[27] P. Hansen, S. Klahn, C. Clausen, G. Much, and K. Witter, J. Appl. Phys. 69, 3194 (1991).

[28] N. H. Duc, T. D. Hien, D. Givord, J. J. M. Franse, and F. R. de Boer, J. Magn. Magn. Mater. 124, 305-311 (1993).

[29] M. Binder, A. Weber, O. Mosendz, G. Woltersdorf, M. Izquierdo, I. Neudecker, J. R. Dahn, T. D. Hatchard, J.-U. Thiele, C. H. Back et al., Phys. Rev. B 74, 134404 (2006).

[30] C. D. Stanciu, A. V. Kimel, F. Hansteen, A. Tsukamoto, A. Itoh, A. Kirilyuk, and T. Rasing, Phys. Rev. B 73, 220402 (2006).

[31] V. N. Krivoruchko, Low Temp. Phys. 40, 42 (2014).

[32] S. Wienholdt, D. Hinzke, and U. Nowak, Phys. Rev. Lett. 108, 247207 (2012).

[33] R. Medapalli, I. Razdolski, M. Savoini, A. R. Khorsand, A. Kirilyuk, A. V. Kimel, T. Rasing, A. M. Kalashnikova, A. Tsukamoto, and A. Itoh, Phys. Rev. B 86, 054442 (2012).

[34] R. Medapalli, I. Razdolski, M. Savoini, A. R. Khorsand, A. M. Kalashnikova, A. Tsukamoto, A. Itoh, A. Kirilyuk, A. V. Kimel, and T. Rasing, Eur. Phys. J. B 86, 183 (2013).

[35] A. Mekkonen, A. R. Khorsand, M. Cormier, A. V. Kimel, A. Kirilyuk, A. Hrabec, L. Ranno, A. Tsukamoto, A. Itoh, and T. Rasing, Phys. Rev. B 87, 180406(R) (2013).

[36] D. A. Garanin, Phys. Rev. B 55, 3050 (1997).

[37] U. Atxitia, O. Chubykalo-Fesenko, J. Walowski, A. Mann, and M. Münzenberg, Phys. Rev. B 81, 174401 (2010).

[38] U. Atxitia and O. Chubykalo-Fesenko, Phys. Rev. B 84, 144414 (2011).

[39] U. Atxitia, P. Nieves, and O. Chubykalo-Fesenko, Phys. Rev. B 86, 104414 (2012).

[40] U. Atxitia, D. Hinzke, and U. Nowak, J. Phys. D: Appl. Phys. 50, 033003 (2017).

[41] O. J. Suarez, P. Nieves, D. Laroze, D. Altbir, and O. ChubykaloFesenko, Phys. Rev. B 92, 144425 (2015).
[42] V. N. Gridnev, J. Phys.: Condens. Matter. 28, 476007 (2016).

[43] I. Radu, C. Stamm, A. Eschenlohr, F. Radu, R. Abrudan, K. Vahaplar, T. Kachel, N. Pontius, R. Mitzner, K. Holldack et al., SPIN 5, 1550004 (2015).

[44] K. Holldack, J. Bahrdt, A. Balzer, U. Bovensiepen, M. Brzhezinskaya, A. Erko, A. Eschenlohr, R. Follath, A. Frisov, W. Frentrup et al., J. Synchrotron Radiat. 21, 1090 (2014).

[45] S. Honda and M. Yoshiyama, Jpn. J. Appl. Phys. 27, 2073 (1988).

[46] M. Mansuripur and M. Ruane, IEEE Trans. Magn. 22, 33 (1986).

[47] K. Chen, D. Lott, F. Radu, F. Choueikani, E. Otero, and P. Ohresser, Phys. Rev. B 91, 024409 (2015).

[48] P. Carra, B. T. Thole, M. Altarelli, and X. Wang, Phys. Rev. Lett. 70, 694 (1993).

[49] B. T. Thole, P. Carra, F. Sette, and G. van der Laan, Phys. Rev. Lett. 68, 1943 (1992).

[50] Y. Teramura, A. Tanaka, B. T. Thole, and T. Jo, J. Phys. Soc. Jpn. 65, 3056 (1996).

[51] Y. Teramura, A. Tanaka, and T. Jo, J. Phys. Soc. Jpn. 65, 1053 (1996).

[52] S. Mangin, C. Bellouard, S. Andrieu, F. Montaigne, P. Ohresser, N. B. Brookes, and B. Barbara, Phys. Rev. B 70, 014401 (2004).

[53] Y. Guan, Z. Dios, D. A. Arena, L. Cheng, and W. E. Bailey, J. Appl. Phys. 97, 10A719 (2005).

[54] A. Agui, M. Mizumaki, T. Asahi, K. Matsumoto, T. Morikawa, J. Sayama, and T. Osaka, J. Phys. Chem. Solids 68, 2148 (2007).

[55] A. Donges, S. Khmelevskyi, A. Deak, R.-M. Abrudan, D. Schmitz, I. Radu, F. Radu, L. Szunyogh, and U. Nowak, Phys. Rev. B 96, 024412 (2017).

[56] C. Illg, M. Haag, and M. Fähnle, Phys. Rev. B 88, 214404 (2013).

[57] M. Haag, C. Illg, and M. Fähnle, Phys. Rev. B 90, 014417 (2014).

[58] M. Fähnle, C. Illg, M. Haag, and N. Teeny, Acta Phys. Pol. A 127, 170 (2015).

[59] C. E. Graves, A. H. Reid, T. Wang, B. Wu, S. de Jong, K. Vahaplar, I. Radu, D. P. Bernstein, M. Messerschmidt, L. Müller et al., Nat. Mater. 12, 293 (2013).

[60] D. J. Higley, K. Hirsch, G. L. Dakovski, E. Jal, E. Yuan, T. Liu, A. A. Lutman, J. P. MacArthur, E. Arenholz, Z. Chen et al., Rev. Sci. Instrum. 87, 033110 (2016).

[61] U. Atxitia, J. Barker, R. W. Chantrell, and O. ChubykaloFesenko, Phys. Rev. B 89, 224421 (2014).

[62] V. N. Krivoruchko, Phys. Rev. B 94, 054434 (2016).

[63] A. J. Schellekens and B. Koopmans, Phys. Rev. B 87, 020407 (2013).

[64] A. M. Kalashnikova and V. I. Kozub, Phys. Rev. B 93, 054424 (2016).

[65] M. S. El Hadri, P. Pirro, C.-H. Lambert, S. Petit-Watelot, Y. Quessab, M. Hehn, F. Montaigne, G. Malinowski, and S. Mangin, Phys. Rev. B 94, 064412 (2016).

[66] A. Baral and H. C. Schneider, Phys. Rev. B 91, 100402 (2015).

[67] M. Sultan, A. Melnikov, and U. Bovensiepen, Phys. Status Solidi B 248, 2323 (2011).

[68] T. Roth, A. J. Schellekens, S. Alebrand, O. Schmitt, D. Steil, B Koopmans, M. Cinchetti, and M. Aeschlimann, Phys. Rev. X 2, 021006 (2012). 
[69] B. Frietsch, R. Carley, M. Gleich, M. Teichmann, J. Bowlan, and M. Wienelt, Jpn. J. Appl. Phys. 55, 07MD02 (2016).

[70] K. C. Kuiper, G. Malinowski, F. Dalla Longa, and B. Koopmans, J. Appl. Phys. 109, 07D316 (2011).

[71] J. H. Mentink, J. Hellsvik, D. V. Afanasiev, B. A. Ivanov, A. Kirilyuk, A. V. Kimel, O. Eriksson, M. I. Katsnelson, and T. Rasing, Phys. Rev. Lett. 108, 057202 (2012).
[72] N. Kazantseva, U. Nowak, R. W. Chantrell, J. Hohlfeld, and A. Rebei, Europhys. Lett. 81, 27004 (2008).

[73] R. Moreno, T. A. Ostler, R. W. Chantrell, and O. ChubykaloFesenko, Phys. Rev. B 96, 014409 (2017).

[74] J. Barker, U. Atxitia, T. A. Ostler, O. Hovorka, O. Chubykalo-Fesenko, and R. W. Chantrell, Sci. Rep. 3, 3262 (2013). 